\title{
Fondaparinux in major orthopedic surgical patients
}

\author{
Roberto Santi $\cdot$ Laura Contino $\cdot$ Simona Gatto $\cdot$ \\ Alessandro Levis
}

Received: 15 January 2010/ Accepted: 28 January 2010/Published online: 2 March 2010

(C) SIMI 2010

S.Capri and co-workers confirmed in an extensive number of patients undergoing major orthopaedic surgery (MOS) in Italy that prophylaxis with fondaparinux was more cost saving than enoxaparin [1]. This result is very important because other economic evaluations show an incremental cost-effectiveness ratio (ICER) in favour of fondaparinux only in short-term thromboprophylaxis, and none of them included Italian data. The authors assessed the overall cost of extended prophylaxis of venous thromboembolism (VTE), calculating the cost of management of each symptomatic VTE episode, taking into account early and late VTE events and other sequelae.

This new evidence suggests more evaluations are needed.

In addition to being more effective than enoxaparin in preventing VTE in patients undergoing MOS, in extended prophylaxis, fondaparinux is cost saving up to 5 years.

In Capri and co-workers' study, the occurrence of heparin-induced thrombocytopenia (HIT) was excluded from the cost-effectiveness analysis of fondaparinux versus enoxaparin. It has been observed that antiPF4 heparin antibodies occur less frequently in thromboprophylaxis with fondaparinux than with low molecular weight heparin (LMWH). Fondaparinux does not cross-react in vitro with HIT antibodies, particularly it does not react with PF4 in such a way as to cause sites for HIT antibody binding [2]. For these reasons the use of fondaparinux in thromboprophylaxis is associated with a lower risk of HIT, and specifically with a negligible risk in patients who may have

R. Santi $(\bowtie) \cdot$ L. Contino $\cdot$ S. Gatto $\cdot$ A. Levis Dept. Oncohematology, Thrombosis Center,

Az. Osp. SS. Antonio e Biagio e C.Arrigo, via Venezia 16, 15100 Alessandria, Italy

e-mail: rsanti@ospedale.al.it recently undergone cardiac surgery or invasive cardiac procedures.

In our opinion, this economic and clinical evaluation suggests that fondaparinux should be considered the standard therapy in patients undergoing MOS, in particular in Italian patients for whom this evaluation was done.

Therefore, we think that new anticoagulant agents in MOS should be tested versus fondaparinux and not versus enoxaparin. This is very important in light of the recent introduction of dabigatran and rivaroxaban [3-5]. They are new effective drugs in patients undergoing MOS, but it is not clear if they are more effective than fondaparinux because at the moment there are no studies that compare these drugs.

Conflict of interest None.

\section{References}

1. Capri S, Ageno W, Imberti D, Palareti G, Piovella F, Scannapieco G, Moia M (2009) Extended prophylaxis of venous thromboembolism with fondaparinux in patients undergoing major orthopedic surgery in Italy: a cost-effectiveness analysis. Intern Emerg Med 5:33-40

2. Warketin TE, Cook RJ, Marder VJ, Sheppard JA, Moore JA, Eriksson BI (2005) Anti-platelet factor 4/heparin antibodies in orthopedic surgery patients receiving antithrombotic prophylaxis with fondaparinux or enoxaparin. Blood 106:3791-3796

3. Agnelli G, Becattini C (2009) New oral anticoagulants: just a new therapeutic option or a real breakthrough? Intern Emerg Med 4:455-457

4. Imberti D, Dall'asta C, Pierfranceschi MG (2009) Oral factor Xa inhibitors for thromboprophylaxis in major orthopedic surgery: a review. Intern Emerg Med 4:471-477

5. Squizzato A, Dentali F, Steidl L, Ageno W (2009) New direct thrombin inhibitors. Intern Emerg Med 4:479-484 